\title{
Bulk Properties of Symmetric Nuclear and Pure Neutron Matter
}

\author{
Khaled Hassaneen $^{1,2}$, Hesham Mansour ${ }^{3}$ \\ ${ }^{1}$ Department of Physics, Faculty of Science, Sohag University, Sohag, Egypt \\ ${ }^{2}$ Department of Physics, Faculty of Science, Taif University, Taif, Saudi Arabia \\ ${ }^{3}$ Department of Physics, Faculty of Science, Cairo University, Gizza, Egypt
}

Received 2013

\begin{abstract}
We study the equation of state (EOS) of symmetric nuclear and neutron matter within the framework of the Brueckner-Hartree-Fock (BHF) approach which is extended by including a density-dependent contact interaction to achieve the empirical saturation property of symmetric nuclear matter. This method is shown to affect significantly the nuclear matter EOS and the density dependence of nuclear symmetry energy at high densities above the normal nuclear matter density, and it is necessary for reproducing the empirical saturation property of symmetric nuclear matter in a nonrelativistic microscopic framework. Realistic nucleon-nucleon interactions which reproduce the nucleon-nucleon phase shifts are used in the present calculations.
\end{abstract}

Keywords: Symmetric Nuclear Matter; Equation of State; Three-Body Force; Symmetry Energy

\section{Introduction}

Many-body calculations which are based on the realistic bare nucleon-nucleon potentials are able to reproduce qualitatively but not quantitatively the saturation properties of symmetric nuclear matter. The saturation points calculated with different approaches implemented with various choices of the nucleon-nucleon $(N N)$ potential lie on the so called Coester line in the energy per particle-density plane, away from the experimentally allowed values [1]. The theoretical predictions give a saturation density sensibly higher than the experimental value $\rho_{0} \approx$ $0.16 \mathrm{fm}^{-3}$ (usually in the range $1.5 \rho_{0}-2 \rho_{0}$ ) and often over bind the nuclear system (up to $25 \%$ ), failing to get close to the empirical binding energy $E_{0} \approx-16 \mathrm{MeV}$. These discrepancies and uncertainties get amplified when calculating the equation of state (EOS) of pure neutron matter, which is necessary for the estimates of key quantities such as the symmetry energy and in general for the description of neutron-rich matter in neutron stars.

Different ways have been developed to obtain predicttions for the properties of nuclear systems. One way is to start from phenomenological models which successfully describe the properties of stable nuclei. A very popular approach along this line is the use of an effective density dependent Skyrme-type interaction [2,3]. Modern Skyrme parameterizations have been developed, which were constrained in their fitting procedures to obtain results for neutron-rich nuclear matter which are compatible to those of microscopic calculations. Also the relativistic mean-field approximation has very successfully been used to describe the properties of stable nuclei [4].

Therefore, there are some of the microscopic approaches, which start from models of the $N N$ interaction, which are adjusted to describe the experimental phase shifts of $N N$ scattering at energies below the pion threshold. The traditional models of such realistic $N N$ interactions like, e.g., the charge-dependent Bonn (CD-Bonn) potential [5] or the Reid 93 or Nijm1 potentials [6]. Such nonperturbative approximations include the Brueckner hole-line expansion with the Brueckner Hartree-Fock (BHF) [1] approximation, the self-consistent evaluation of Green's function using the T-matrix or G-matrix approximation [7-12] (SCGF) and also variational approaches using correlated basis functions [13].

In the present work we implement the self consistent G-matrix scheme with three different realistic $N N$ potentials (CD-Bonn, Nijm1 and Reid 93) plus a density-dependent contact interaction to achieve the empirical saturation.

\section{BHF for Symmetric Nuclear Matter}

In the BHF approximation, the nuclear matter total energy $E_{A}$ is obtained from the Brueckner G-matrix, $G(\omega)$, according to the equation:

$$
\mathrm{E}_{\mathrm{A}}=\sum_{\mathrm{k}_{1}<\mathrm{k}_{\mathrm{F}}} \frac{\hbar^{2} \mathrm{k}_{1}^{2}}{2 \mathrm{~m}}+\frac{1}{2} \sum_{\mathrm{k}_{1}, \mathrm{k}_{2}<\mathrm{k}_{\mathrm{F}}}\left\langle\mathrm{k}_{1} \mathrm{k}_{2}\left|\mathrm{G}\left(\mathrm{e}_{\mathrm{k}_{1}}+\mathrm{e}_{\mathrm{k}_{2}}\right)\right| \mathrm{k}_{3} \mathrm{k}_{4}\right\rangle \mathrm{a}(1)
$$


with $\left|\mathrm{k}_{1} \mathrm{k}_{2}\right\rangle_{\mathrm{a}}=\left|\mathrm{k}_{2} \mathrm{k}_{1}\right\rangle$, i.e., the subscript a indicates antisymmetrization of the matrix elements. Here $\mathrm{k}_{\mathrm{F}}$ is the Fermi momentum, the summation over the momenta $\mathrm{k}_{\mathrm{i}}$ include spin and isospin variables. The single particle energies $e_{k}$, appearing in the entry energy of the G-matrix, are given by:

$$
\mathrm{e}(\mathrm{k})=\frac{\hbar^{2} \mathrm{k}^{2}}{2 \mathrm{~m}}+\mathrm{U}(\mathrm{k})
$$

where, the single particle potential $\mathrm{U}(\mathrm{k})$ is determined by the self-consistent equation:

$$
\mathrm{U}(\mathrm{k})=\sum_{\mathrm{k}^{\prime} \mathrm{k}_{\mathrm{F}}}\left\langle\mathrm{kk}^{\prime}\left|\mathrm{G}\left(\mathrm{e}_{\mathrm{k}_{1}}+\mathrm{e}_{\mathrm{k}_{2}}\right)\right| \mathrm{kk}^{\prime}\right\rangle
$$

The self-consistency is coupled with the integral equation for the G-matrix, i.e., in the BHF approach $\mathrm{G}(\omega)$ is obtained by solving the Bethe-Goldstone equation:

$$
\begin{aligned}
& \left\langle\mathrm{k}_{1} \mathrm{k}_{2}|\mathrm{G}(\omega)| \mathrm{k}_{3} \mathrm{k}_{4}\right\rangle=\left\langle\mathrm{k}_{1} \mathrm{k}_{2}|\mathrm{v}| \mathrm{k}_{3} \mathrm{k}_{4}\right\rangle+ \\
& \sum_{\mathrm{k}_{3}^{\prime} \mathrm{k}_{4}^{\prime}}\left\langle\mathrm{k}_{1} \mathrm{k}_{2}|\mathrm{v}| \mathrm{k}_{3}^{\prime} \mathrm{k}_{4}^{\prime}\right\rangle \frac{\left(1-\Theta_{\mathrm{F}}\left(\mathrm{k}_{3}^{\prime}\right)\right)\left(1-\Theta_{\mathrm{F}}\left(\mathrm{k}_{4}^{\prime}\right)\right)}{\omega-\mathrm{e}_{\mathrm{k}_{3}^{\prime}}-\mathrm{e}_{\mathrm{k}_{3}^{\prime}}}(4) \\
& \left\langle\mathrm{k}_{3}^{\prime} \mathrm{k}_{4}^{\prime}|\mathrm{G}(\omega)| \mathrm{k}_{3} \mathrm{k}_{4}\right\rangle
\end{aligned}
$$

where, $\Theta_{\mathrm{F}}(\mathrm{k})=1$ defining the step function for $\mathrm{k}<\mathrm{k}_{\mathrm{F}}$ and is zero otherwise and $\omega$ denotes the starting energy. The product $Q\left(\mathrm{k}, \mathrm{k}^{\prime}\right)=\left(1-\Theta_{\mathrm{F}}(\mathrm{k})\right)\left(1-\Theta_{\mathrm{F}}\left(\mathrm{k}^{\prime}\right)\right)$, appearing in the kernel of Equation (4), enforces the scattered momenta to lie outside the Fermi sphere and it is commonly referred to as the "Pauli operator". In the case of the angle-aver-age of Pauli operator this energy is given as, [14]:

$$
\begin{aligned}
& \mathrm{E}_{\mathrm{A}}=\frac{3 \mathrm{k}_{\mathrm{F}}^{2}}{10 \mathrm{~m}}+\frac{6}{\mathrm{k}_{\mathrm{E}}^{3}} \sum_{\mathrm{TS} !}(2 \mathrm{~T}+1)(2 \mathrm{I}+1) \int_{0}^{\mathrm{h}_{\mathrm{F}}} \mathrm{dkk}^{2}
\end{aligned}
$$

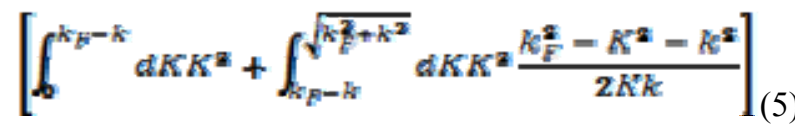$$
\text { (KIJ) } \left.\left.G_{S T}(\omega, K) \mid R E\right)\right\rangle
$$

If one assumes that the potential $U(k)$, or equivalently the single particle energy $e(k)$, has approximately a quadratic form

$$
\mathrm{e}(\mathrm{k}) \approx \mathrm{e}_{0}+\frac{\hbar^{2} \mathrm{k}^{2}}{2 \mathrm{~m}^{*}}
$$

where, $\mathrm{e}_{0}$ is the zero point energy. Then one can calculate the potential, at each iteration step, in few points only and interpolate the obtained values with a parabola. The approximation of Equations (6) is usually called the effective mass approximation, since then the spectrum has the same shape as the free one but with an effective mass $m^{*}$. From Equations (2) and (6) the effective mass $m^{*}$ can be evaluated from the slope of $U(k)$ at the Fermi momentum [15],

$$
\frac{\mathrm{m}^{*}}{\mathrm{~m}}=\left[1+\frac{\mathrm{m}}{\hbar^{2} \mathrm{k}} \frac{\mathrm{dU}}{\mathrm{dk}}\right]_{\mathrm{k}=\mathrm{k}_{\mathrm{F}}}^{-1}
$$

In the present work one may introduce a Skyrme effective interaction density dependent term [2] in addition to the BHF potential.

$$
\mathrm{V}\left(\mathrm{r}_{1}, \mathrm{r}_{2}\right)=\sum_{\mathrm{i}=1}^{4} \mathrm{t}_{\mathrm{i}}\left(1+\mathrm{x}_{\mathrm{i}} \mathrm{P}_{\sigma}\right) \rho^{\alpha} \mathrm{i}_{\delta}\left(\mathrm{r}_{1}-\mathrm{r}_{2}\right) \text {. }
$$

This is a two-body density dependent potential which is equivalent to three-body interaction. Where $t_{i}$ and $x_{i}$ are interaction parameters, $P_{\sigma}$ is the spin exchange operator, $\rho$ is the density, $\mathbf{r}_{1}$ and $\mathbf{r}_{2}$ are the position vectors of the particle (1) and particle (2) respectively and $\alpha_{i}=$ $(1 / 3,2 / 3,1 / 2$ and 1$)$. The parameter $\alpha$ is added to this term to soften the density dependence which could otherwise lead to too high incompressibility for nuclear matter. In order to reproduce the empirical saturation point of symmetric nuclear matter, we have to fit the parameters $t_{i}$. The parameters would be then useful just to give a more quantitative estimate of the needed correction to the BHF results, (which do not reproduce the correct position and value of the saturation point of the nuclear matter EOS). The parameters $\mathrm{x}_{\mathrm{i}}$ are determined by fitting with the experimental symmetry energy. In actual fact we attempted to take one, two, three and four terms of the above equation to fit the data and we found that a satisfactory fit is obtained around the empirical point of the EOS using only two terms of the above summation with $\alpha_{i}=1 / 3$ and $2 / 3$ only. The results for these fitting parameters are listed in Table 1.

\section{Results and Discussion}

The energy per particle (E/A) as a function of density $\rho$ in $\mathrm{fm}^{-3}$ for nuclear matter is shown in Figure 1. Upper panel represents the symmetric nuclear matter and pure neutron matter is shown by lower panel. The calculations have been performed using BHF approach with and without a density dependent interaction as $3 \mathrm{BF}$ with

Table 1. Parameters $t_{i}$ and $x_{i}$ defining a two-body density dependent potential of Eq. (9) as obtained for the fit to the saturation point $\rho_{0}=0.17 \mathrm{fm}^{-3} ; \mathrm{E} / \mathrm{A}=-16 \mathrm{MeV}$ for different potentials.

\begin{tabular}{ccccc}
\hline \multirow{2}{*}{ Potential } & \multicolumn{4}{c}{ Continuous choice } \\
\cline { 2 - 5 } & $\mathrm{t}_{1}$ & $\mathrm{t}_{2}$ & $\mathrm{x}_{1}$ & $\mathrm{x}_{2}$ \\
\hline CD-Bonn & -656.5 & 1197.8 & -0.2991 & -0.7721 \\
Nijm1 & -673.2 & 1156 & -0.5146 & -0.9705 \\
Reid 93 & -782.4 & 1176.2 & -0.4472 & -1.0043 \\
\hline
\end{tabular}



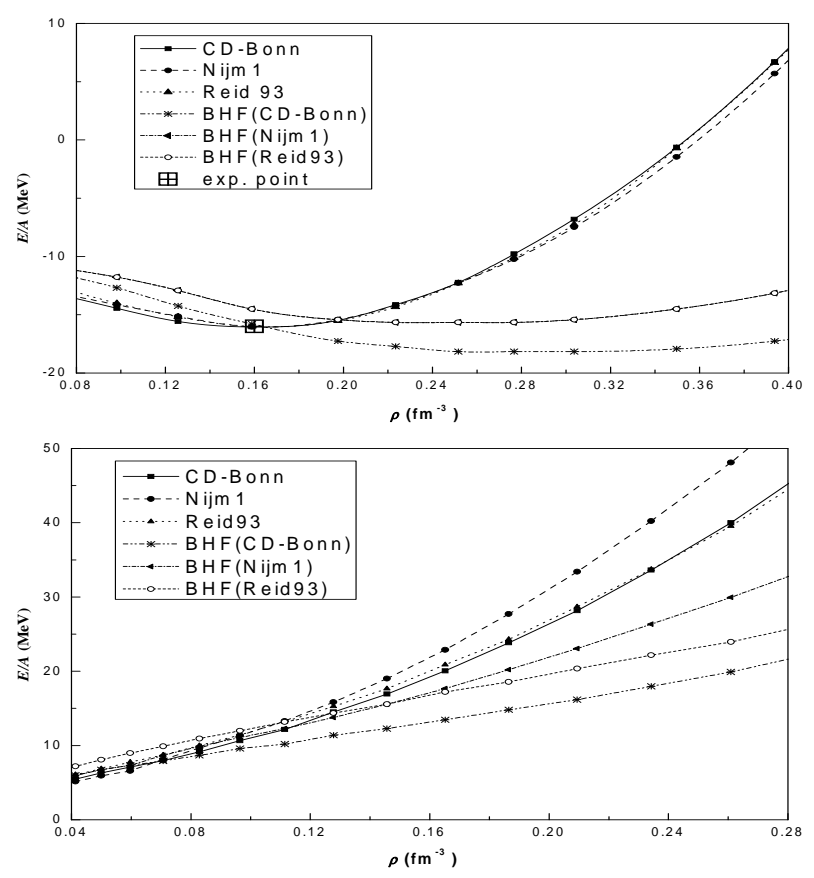

Figure 1. E/A in MeV for symmetric nuclear matter (upper panel) and pure neutron matter (lower panel) using different potentials at $(T=0)$ as a function of density using different potentials for continuous choice of the auxiliary potential.

Adjustable parameters given in Table 1 using different potentials. The relevance of the three-body forces is immediately seen from the shift of the saturation point to $\rho_{0}$ $\approx 0.17 \mathrm{fm}^{-3} ; \mathrm{E} / \mathrm{A} \approx-16 \mathrm{MeV}$, close to the empirical one. The effect of these forces is also very small at low densities and becomes larger at increasing densities, where a much stronger repulsion is apparent. In symmetric nuclear matter the is-ovector components of the interaction does not contribute to the energy per nucleon and therefore, the various in-teractions considered here are expected to give similar EOS up to moderate values of the density.

For more enhancement of the EOS of symmetric nuclear matter, we have added more parameters as defined in Eqution (8) choosing values of $\alpha_{\mathrm{i}}=(1 / 3,2 / 3,1 / 2$ and 1) and fitting the parameters $t_{i}$ and $x_{i}$. The results for these fitting parameters are listed in Table 2 and the corresponding energy versus density curves are displayed in Figure 2 using CD-Bonn interaction. The suggestion was very general to fit the properties of the nuclear and neutron matter. Therefore we take a number of points which is equal to the number of parameters to proceed with the fitting procedure. We started with two terms of the potential i.e.4-parameters. In this case we took the saturation points for E/A and the pressure besides two more points of the experimental data of the symmetry energy. Good fit was observed near the minimum of the EOS and the symmetry energy as well. We gave an example if one uses four terms of the potential with 8-parameters which shows a better fit for $\mathrm{E} / \mathrm{A}$ and the pressure over a wider densities scale. In this case we took two more points on both the E/A and pressure data to obtain the rest of the parameters. Our observation was that a complete and good fit was obtained for the nuclear matter data but satisfactory fit for the neutron matter data.

Our results are compared with the results obtained by Freidman and Pandharipande (F and P) using the variational calculations [16]. One sees that a good agreement is obtained for a wide range of $\rho$. Also, the present calculations are compared with the microscopic calculations with the BHF approach supplemented by three-body forces using CD-Bonn potential by Baldo and Shaban [17], self-consistent Green's function (SCGF) approach with an exact treatment of Pauli operator [18]. As expected, the three-body force or a simple two-body density dependent term, like as Equation (8), shifts the minimum of each curve towards the empirical saturation point. The softest EOS for symmetric matter among those approaches which fit the empirical saturation point is provided by the BHF approximation. Also, the steepness of the EOS at higher densities can depend of course on the particular three-body forces introduced in the calculations, but the region around saturation is expected to be insensitive to the details of the force used, since they are constrained to reproduce this region.

Table 2. All parameters $t_{i}$ and $x_{i}$ defining a two-body density dependent potential of Equation (8) as obtained for the fit to the saturation point $\rho_{0}=0.17 \mathrm{fm}^{-3} ; \mathrm{E} / \mathrm{A}=-16 \mathrm{MeV}$ for CD- Bonn potential.

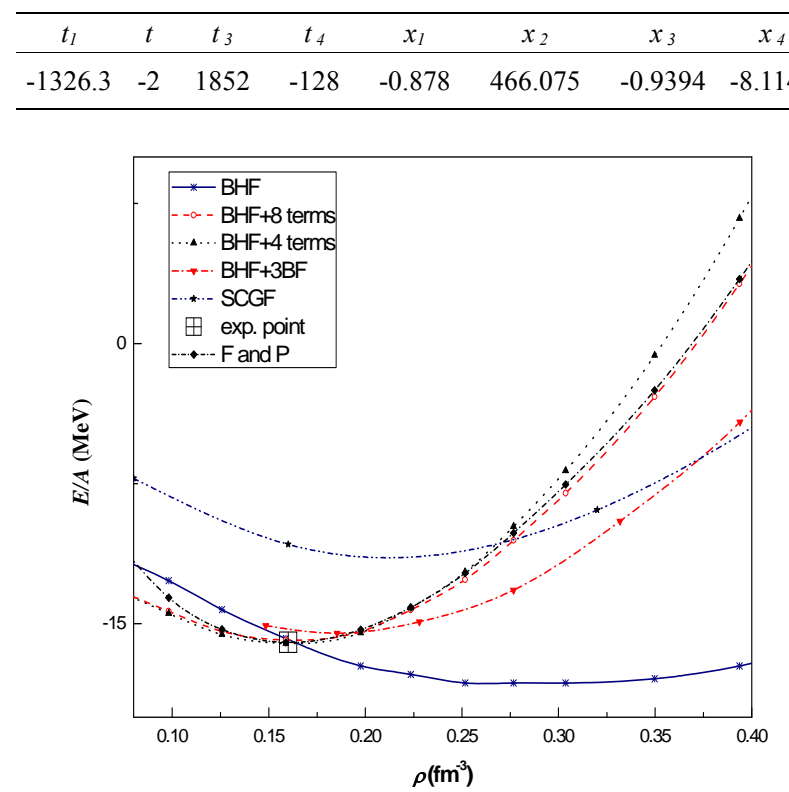

Figure 2. E/A in MeV for symmetric nuclear matter at $(T=$ 0) as a function of density using CD-Bonn potential for continuous choice in comparison with different approaches. 
An important quantity in determining the equation of state of isospin asymmetric nuclear matter is the symmetry energy. The symmetry energy is defined from the energy per nucleon $\mathrm{E} / \mathrm{A}$ as follows

$$
\mathrm{E}_{\text {sym }}(\rho)=\frac{1}{2}\left[\frac{\partial^{2} \mathrm{E}_{\mathrm{A}}(\rho, \alpha)}{\partial \alpha^{2}}\right]_{\alpha=0}
$$

Both $\rho_{\mathrm{n}}$ and $\rho_{\mathrm{p}}$ are the neutron and proton densities in Asymmetric Nuclear Matter (ANM) and $\rho=\rho_{n}+\rho_{p}$ is the total density of asymmetric nuclear matter. It is well established [19-20] that the binding energy per nucleon $\mathrm{E}_{\mathrm{A}}$ fulfills the simple $\mathrm{a}^{2}$-law not only for a«1 as assumed in the empirical nuclear mass formula [21], but also in the whole asymmetry range. The $\mathrm{a}^{2}$-law of the EOS of ANM at any isospin asymmetry leads to two important consequences. This enables us to calculate the symmetry energy $E_{\text {sym }}$ in terms of the difference between the binding energy of pure neutron matter $\mathrm{E}_{\mathrm{A}}(\rho, 1)$ and that of symmetric nuclear matter $\mathrm{E}_{\mathrm{A}}(\rho, 0)$, i.e.,:

$$
\mathrm{E}_{\text {sym }}(\rho)=\mathrm{E}_{\mathrm{A}}(\rho, 1)-\mathrm{E}_{\mathrm{A}}(\rho, 0),
$$

But one would refrain from applying it at very high density. The results of our calculation for the symmetry energy as a function of baryonic density in terms of the Fermi momentum $\mathrm{k}_{\mathrm{F}}$ are depicted in Figure 3.
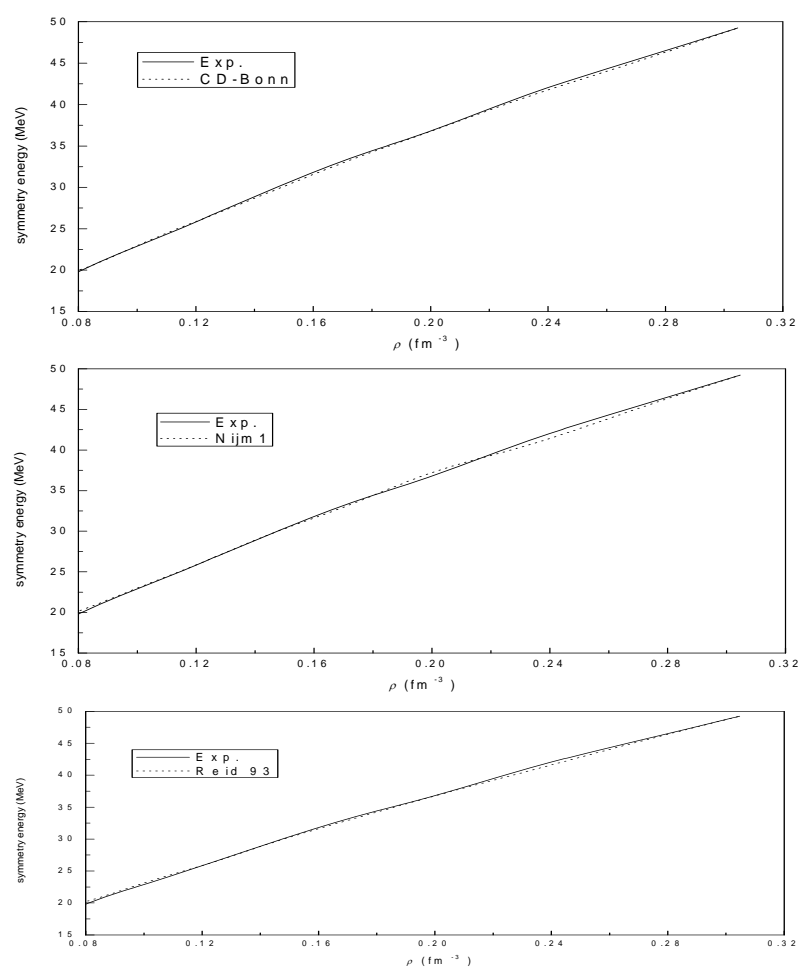

Figure 3. The symmetry energy in $\mathrm{MeV}$ as a function of density in $\mathrm{fm}^{-3}$ in comparison with the experimental data [22] using different potentials for the continuous choice.

In Figure 3 for continuous choice, symmetry energies in $\mathrm{MeV}$ are plotted against the density $\rho$ in $\mathrm{fm}^{-3}$ in comparison with the experimental data [22] represented by solid line using the CD-Bonn potential, the Nijm1 potential, and the Reid 93 potential with dot lines from Figure 3. It is observed that when the density of nuclear matter increases the symmetry energy of the system increases in agreement with the experimental results. For all potentials at the saturation density $\left(\rho_{0}=0.16 \mathrm{fm}^{-3}\right)$ it is found that the nuclear symmetry energy is around the empirical value, $32 \mathrm{MeV}$.

The nuclear incompressibility $\kappa_{0}$ characterizes the stiffness of the EOS of symmetric nuclear matter. The experimental value of the incompressibility of nuclear matter at its saturation density $\rho_{0}$ has been determined to be $210 \pm 30 \mathrm{MeV}$ [23]. The incompressibility $\kappa_{0}$ at the saturation point $\rho_{0}$ is given by

$$
\kappa_{0}=\left.\mathrm{k}_{\mathrm{F}}^{2} \frac{\partial^{2}(\mathrm{E} / \mathrm{A})\left(\mathrm{k}_{\mathrm{F}}\right)}{\partial \mathrm{k}_{\mathrm{F}}^{2}}\right|_{\mathrm{k}=\mathrm{k}=\mathrm{F}}=\left.9 \rho^{2} \frac{\partial^{2}(\mathrm{E} / \mathrm{A})(\rho)}{\partial \rho^{2}}\right|_{\rho=\rho_{0}} .
$$

The results for the incompressibility for the present calculations at the saturation points are 208.5, 184.6 and 228.2 MeV for the CD-Bonn, Nijm1 and Reid93 potentials.

\section{Summary}

The bulk properties of symmetric nuclear matter are computed such as the equation of state, nuclear matter symmetry energy and incompressibility as a function of the density. The calculations of the above properties for symmetric nuclear matter are made by using BHF interaction + two-body density dependent Skyrme interaction which is equivalent to three-body interaction. Modern $N N$ interactions such as the CD-Bonn potential, the Nijm1 potential, and the Reid 93 potential are used in order to analyze the dependence of the results on the nuclear interaction. Good values are obtained for the incompressibility showing the stiffness of each potential with respect to the others. The symmetry energy also shows a good agreement with the experimental data. We conclude that the BHF theory in addition to our suggested contact interaction is able to produce the experimental saturation point for the equation of state. Among the different choices of the sets of parameters $t_{i}$ and $x_{i}$ best results were obtained for the set of parameters given here. Two terms are used only in our suggested potential. One can add other terms to calculate other physical quantities. In fact terms with other values of the parameter $\alpha 4 / 3,5 / 3,3 / 2$ and 2 may be envisaged in order to get a fit with the inclusion of neutron matter properties. 


\section{REFERENCES}

[1] Kh. S. A. Hassaneen, H. M. Abo-Elsebaa, E. A. Sultan and H. M. M. Mansour, "Nuclear Binding Energy and Symmetry Energy of Nuclear Matter with Modern Nucleon-Nucleon Potentials," Annals of Physics, Vol. 326, No. 3, 2011, pp. 566-577. doi:10.1016/j.aop.2010.11.010

[2] D. Vautherin and D. M. Brink, "Hartree-Fock Calculations with Skyrme's Interaction. I. Spherical Nuclei," Physical Review C, Vol. 5, No. 3, 1972, pp. 626-647. doi:10.1103/PhysRevC.5.626

[3] J. R. Stone and P. -G. Reinhardt, "The Skyrme Interaction in Finite Nuclei and Nuclear Matter," Progress Particle Nuclear Physics, Vol. 58, No. 2, 2007, pp. 587-657. doi:10.1016/i.ppnp.2006.07.001

[4] D. Vretenar, A. Afanasjev, G. A. Lalazissis and P. Ring, "Relativistic Hartree-Bogoliubov Theory: Static and Dynamic Aspects of Exotic Nuclear Structure," Physics Reports, Vol. 409, No. 3-4, 2005, pp. 101-259. doi:10.1016/j.physrep.2004.10.001

[5] R. Machleidt, "High-Precision, Charge-Dependent Bonn Nucleon-Nucleon Potential," Physical Review C, Vol. 63, 2001, pp. 024001 (32 pages). doi:10.1103/PhysRevC.63.024001

[6] V. G. J. Stoks, R. A. M. Klomp, C. P. F. Terheggen and J. J. de Swart, "Construction of High-Quality NN Potential," Physical Review C, Vol. 49, 1994, pp. 2950-2962. doi:10.1103/PhysRevC.49.2950

[7] W. H. Dickhoff and C. Barbieri, "Self-Consistent Green' s Function Method for Nuclei and Nuclear Matter," Progress in Particle and Nuclear Physics, Vol. 52, No. 24, pp. 377-496. doi:10.1016/j.ppnp.2004.02.038

[8] Y. Dewulf, W. H. Dickhoff, D. Van Neck, E. R. Stoddard and M. Waroquier, "Saturation of Nuclear Matter and Short-Range Correlations," Physical Review Letters, Vol. 90, 2003, pp. 152501(4 pages). doi:10.1103/PhysRevLett.90.152501

[9] T. Frick, K. Gad, H. Müther and P. Czerski, Nuclear Self-Energy and Realistic Interactions. Physical Review $C$, Vol. 65, 2002, pp. 034321(13 pages). doi:10.1103/PhysRevC.65.034321

[10] T. Frick, Kh. S. A. Hassaneen, D. Rohe and H. Müther, "Spectral Function at High Missing Energies and Momenta," Physical Review C, Vol. 70, 2004, pp. 024309(5 pages). doi:10.1103/PhysRevC.70.024309

[11] Kh. S. A. Hassaneen and H. Müther, "Correlations and Spectral Functions In Asymmetric Nuclear Matter," Physical Review C, Vol. 70, 2004, pp. 054308 (5 pages).

\section{doi:10.1103/PhysRevC.70.054308}

[12] Kh. Gad and Kh. S. A. Hassaneen, "Equation of State for Neutron-Rich Matter with Self-Consistent Green Function Approach," Nuclear Physics A, Vol. 793, No. 1-4, 2007, pp. 67-78. doi:10.1016/j.nuclphysa.2007.06.015

[13] Akmal and V. R. Pandharipande, "Spin-Isospin Structure and Pion Condensation in Nucleon Matter," Physical Review C, Vol. 56, 1997, pp. 2261-2279. doi:10.1103/PhysRevC.56.2261

[14] M.I. Haftel and F. Tabakin, "Nuclear Saturation and the Smoothness of Nucleon-Nucleon Potentials," Nuclear Physics A, Vol. 158, No. 1, 1970, pp. 1-42. doi:10.1016/0375-9474(70)90047-3

[15] C. Mahaux and R. Sartor, "Single-Particle Motion in Nuclei," Advances in Nuclear Physics, Vol. 20, 1991, pp. 1-223. doi:10.1007/978-1-4613-9910-0_1

[16] B. Friedman and V. R. Pandharipande, "Hot and Cold, Nuclear and Neutron Matter," Nuclear Physics A, Vol. 361, No. 2, 1981, pp. 502-520. doi:10.1016/0375-9474(81)90649-7

[17] M. Baldo and A. E. Shaban, "Dependence of the Nuclear Equation of State On Two-Body and Three-Body Forces," Physical Letters B, Vol. 661, No. 5, 2008, pp. 373-377. doi:10.1016/j.physletb.2008.02.040

[18] H. Mansour, Kh. Gad and Kh. S. A. Hassaneen, "Self-Consistent Green Function Calculations for Isospin Asymmetric Nuclear Matter. Prog," Progress of Theoretical Physics, Vol. 123, No. 4, 2010, pp. 687-700. doi:10.1143/PTP.123.687

[19] K. Gad and K. S. A. Hassaneen,. "Equation of State for Neutron-Rich Matter with Self-Consistent Green Function Approach," Nuclear Physics A, Vol. 793, No. 1-4, 2007, pp. 67-78. doi:10.1016/j.nuclphysa.2007.06.015

[20] K. Hassaneen and K. Gad, "The Nuclear Symmetry Energy in Self-Consistent Green-Function Calculations," Journal of The Physical Society of Japan, Vol. 77, 2008, pp. 084201-084206. doi:10.1143/JPSJ.77.084201

[21] P. E. Haustein, “An Overview of The 1986-1987 Atomic Mass Predictions," Atomic Data Nuclear Data Tables, Vol. 39, No. 2,1988, pp. 185-200. doi:10.1016/0092-640X(88)90019-8

[22] S. J. Yennello, D. V. Shetty and G. A. Souliotis, nucl-ex/ 0601006v3.

[23] J. P. Blaizot, "Nuclear Compressibilities," Physics Reports, Vol. 64, No. 4, 1980, pp. 171-248. doi:10.1016/0370-1573(80)90001-0 